A N N A L E S Annales de Bretagne et des Pays de l'Ouest

\title{
La demeure seigneuriale dans l'espace Plantagenêt : salles, chambres et tours
}

\section{Marie Casset}

\section{(2) OpenEdition}

1 Journals

Édition électronique

URL : http://journals.openedition.org/abpo/2792

DOI : $10.4000 /$ abpo. 2792

ISBN : 978-2-7535-3513-8

ISSN : 2108-6443

Éditeur

Presses universitaires de Rennes

Édition imprimée

Date de publication : 30 juin 2014

Pagination : 169-172

ISBN : 978-2-7535-3511-4

ISSN : 0399-0826

\section{Référence électronique}

Marie Casset, "La demeure seigneuriale dans l'espace Plantagenêt: salles, chambres et tours ", Annales de Bretagne et des Pays de l'Ouest [En ligne], 121-2 | 2014, mis en ligne le 30 juin 2014, consulté le 23 septembre 2020. URL : http://journals.openedition.org/abpo/2792 ; DOI : https://doi.org/10.4000/ abpo. 2792 


\section{Comptes rendus}

MEIRION-Jones, Gwyn (dir.), La demeure seigneuriale dans l'espace Plantagenêt : salles, chambres et tours, Rennes, PUR, coll. "Art et société ", 2013, 485 p.

Le gros ouvrage que nous proposent les Presses universitaires de Rennes et un groupe de travail informel franco-anglais sur l'habitat seigneurial ( $\mathrm{Xl}^{\mathrm{e}}-\mathrm{XV}^{\mathrm{e}}$ siècles) mérite toute notre attention pour la masse d'informations délivrées sur un sujet qui a connu un sérieux renouveau depuis une quarantaine d'années sous l'impulsion notable des chercheurs britanniques qui ont fait de la Bretagne (Meirion-Jones) et de la Normandie (Impey) un champ de recherche renouvelé.

Les 485 pages du volume sont consacrées à un avant-propos (G. Meirion-Jones), une introduction (G. Meirion-Jones, P. Garrigou Grandchamp, E. Impey, M. Jones, C. Laurent, D. Shewan), 22 contributions, un index des noms de personnes (6 pages), un index des lieux (22 pages), un index thématique (4 pages). L'ampleur de l'index des lieux est une première bonne illustration de la richesse des données et des sites convoqués pour les démonstrations depuis l'Ecosse jusqu'aux Pyrénées. Les 22 auteurs appartiennent à un groupe de travail informel créé dans les années 1980 sous l'impulsion de G. Meirion-Jones, dont les membres se retrouvent annuellement pour des séminaires et nous découvrons ici l'état de leurs recherches en Angleterre (Dixon, Meirion-Jones), Bretagne (Meirion-Jones, Jones, Guibal), Normandie (NicolasMéry, Impey), Anjou et Touraine (Crépin-Leblond, Litoux, Carré, Hunot), Aquitaine et ses marges (Joineau, Séraphin), Maine (Meuret, Bouvet, Garrigou-Grandchamp, Gautier, Scheffer, Favre), Chartrain (Audebrand). Outre ces données régionales, l'ouvrage offre une étude sur les logis et les meubles (Nassiet) et deux articles de synthèse : la maison urbaine dans le grand Ouest (Garrigou Grandchamp), les mutations des logis en France entre 1350 et 1450 (Salamagne).

Nous avons affaire ici à des chercheurs confirmés de toutes origines : historiens et historiens de l'art, archéologues professionnels, docteurs et doctorants en histoire de l'art et archéologie, responsables de services et de sites patrimoniaux, architectes, archivistes, maçon et dendrochronologues. Nombre d'études de sites sont issues de travaux universitaires (DEA, Masters, thèses en cours) des universités de Tours, Le Mans, Paris I. Ces auteurs aux compétences variées et sûres font de ce volume, outre les conclusions architecturales, un très large et savant éventail des méthodes actuelles de travail pour la datation, la restitution et l'évolution des structures à vocation résidentielle, sur des bâtiments encore en élévation et les charpentes en place; méthodes qui conjuguent archéologie du bâti, datation par dendrochronologie, histoire de l'art et sources écrites (archives privées et publiques), cartographie et iconographie modernes et contemporaines. Certaines contributions montrent avec efficacité que, sur nombre de sites manoriaux déclassés depuis plus ou moins longtemps, des bâtiments devenus granges, étables et souvent en mauvais état ou très modifiés, sont les vestiges des logis nobles primitifs (salle basse à galerie du Xve siècle dans l'Avranchin, manoirs du Maine).

L'archéologie sédimentaire n'est convoquée que dans le travail de J.-C. Meuret qui illustre la continuité possible de l'implantation de certains sites élitaires fos- 
soyés depuis au moins l'époque carolingienne et que la représentation et la mise en scène du pouvoir y jouent un rôle essentiel. Cette très petite place réservée à l'archéologie sédimentaire ne reflète pas franchement ses apports à notre connaissance de l'habitat aristocratique dans l'espace concerné.

L'ouvrage est abondamment illustré : cartes de localisation des sites, cartes et schémas de distribution des structures de charpente en Angleterre, plans, photos, schémas de restitution des éléments disparus. Si la grande majorité des photos est d'excellente qualité, on regrette que certaines, visiblement anciennes, ne portent pas de date; quant aux photos du palais des comtes du Maine au Mans, le rendu flou ne rend pas hommage à l'importance du sujet, aux démonstrations et conclusions de l'auteur.

Le titre et son sous-titre ne rendent pas non plus véritablement compte du contenu très large de l'ouvrage. En effet, alors que l'intitulé " demeure seigneuriale " laisse entendre que nous aurions à faire avec des habitats de la petite et moyenne aristocratie, châtelaine ou non, cinq contributions nous emmènent dans un univers autrement huppé, princier (comtes du Maine, archevêques de Tours, ducs de Normandie, roi René), voire difficile à imaginer comme logis seigneurial (moulins fortifiés du sud-ouest). La contribution sur les maisons urbaines médiévales du grand Ouest (Garrigou Grandchamp) pourrait paraître franchement hors du sujet; mais au-delà d'une belle synthèse inédite sur ce thème pour la région considérée, les données disponibles conduisent à considérer que la structuration verticale des maisons de pierre reproduit celle de l'étage habitable des logis nobles (salle haute chauffée et largement éclairée sur rez-de-chaussée à usage domestique ou commerçant) et mériterait un travail de métrologie.

On relève aussi dans le cadre Plantagenêt, de l'Ecosse aux Pyrénées, une certaine disproportion dans les représentations des provinces qui ont été tenues à des degrés plus ou moins étroits par les Plantagenêt. On aurait peut-être attendu une mise en perspective " Plantagenêt " si elle existe. S'il n'y a pas de " style " Plantagenêt pour les palais épiscopaux (Crépin-Leblond), il existe pour le site de Moulins à SaintRémy-du-Val dans la Sarthe (Favre) et pour les tours à contreforts du Quercy où est décelée une esthétique parlante "Plantagenêt " (Séraphin). Sans tomber dans une arithmétique qui n'aurait pas grand intérêt, on relève l'absence de régions qui ont pesé lourd dans l'espace Plantagenêt (Poitou, Aquitaine) et la faible présence de la Normandie (manoirs des XIV ${ }^{\mathrm{e}} \mathrm{xVI} \mathrm{I}^{\mathrm{e}}$ siècles de l'Avranchin, grande salle des ducs à Caen), alors qu'il existe de nombreux travaux récents d'inventaire et d'analyse.

Les nécessités éditoriales de la réalisation d'un fort volume expliquent sans doute les reprises de travaux déjà publiés par ailleurs (manoirs en Bretagne, origines et débuts du manoir) mais la majorité des contributions sont des travaux inédits particulièrement bien venus dans leurs démonstrations et conclusions où la ligne directrice s'articule autour de l'observation de l'état primitif et de l'évolution des deux espaces résidentiels des habitats aristocratiques : la salle d'apparat/ aula, la chambre/chamber-block/camera. Les problématiques et grilles de lecture sont bien connues : grandes salles d'apparat à l'étage ou de plain-pied sous charpente avec ou sans bas-côtés; quelle configuration pour la camera/chambre dans des bâtiments indépendants, accolés ou sous un même toit? quelles mutations structurales lorsque les modes de vie évoluent vers plus d'intimité et de confort (Salamagne, Litoux) - chambre de parement, chambre de retrait, retrait - par de nouvelles constructions ou l'insertion de planchers? concentration des espaces de vie dans un même bloc structuré de part et d'autre d'une salle de plain-pied sous charpente (Meirion Jones, Nicolas-Méry).

Il faut souligner le grand intérêt scientifique et documentaire des contributions sur des sites remarquables pour lesquels on attendait de nouvelles démarches intel- 
lectuelles. E. Impey et J. Mc Neill justifient, comparaisons stylistiques à l'appui, la datation (fin Xle siècle) et la structuration (hall de plain-pied sous charpente) de la salle dite de l'Echiquier à Caen qui serait devenue un modèle à imiter pour les élites; $N$. Gautier nous donne la première étude architecturale sérieuse du palais des comtes du Maine au Mans dans ses composantes et leur évolution; T. CrépinLeblond ajoute au corpus des palais épiscopaux étudiés ces dernières années, la grande salle du palais de Tours qui ne diffère pas, dans la chronologie de la construction romane et dans sa structuration, des grandes salles épiscopales au nord de la Loire qu'il connaît bien.

Les synthèses régionales inédites (Anjou, Maine), dressées à partir de monographies déjà publiées, établissent un très intéressant état de l'évolution de l'architecture seigneuriale (Carré, Litoux, Hunot, Bouvet, Garrigou Grandchamp). La grande salle dont la vocation primitive, par son ampleur et son relatif isolement, contribue à la mise en scène du pouvoir du maître des lieux, disparaît visuellement à partir du $\mathrm{Xv}^{\mathrm{e}}$ siècle; on y implante un plafond pour créer un étage sous comble et dans les logis neufs elle est insérée dans un bloc unique qui concentre tous les espaces résidentiels (salle et chambres, cuisine). En Angleterre, la relativement bonne conservation des charpentes et des pans de bois des grandes salles comme des granges, permet à P. Dixon d'établir les périodes d'émergence, les aires de diffusion des formes et des savoir-faire des œuvres de charpenterie (cruck, crown post).

Six contributions nous donnent onze monographies de sites manoriaux (Sarthe, Avranchin, Eure-et-Loir, Indre-et-Loire) où les dispositions et l'évolution des espaces résidentiels sont scrutées par l'archéologie du bâti et la dendrochronologie; certaines structures sont avec justesse comparées avec des sites extérieurs aux régions considérées (Normandie, Angleterre) en particulier pour ce qui concerne l'adoption des grandes salles de plain-pied sous charpente à bas-côtés. Ils sont aussi l'occasion de faire entrer dans l'ouvrage que nous considérons des programmes propres à tous les niveaux de l'aristocratie, qui n'ont pas été privilégiés comme tels dans l'ouvrage, les logis-porche (Piron, Scheffer). Cette variante du logis avec salle et chambre en enfilade à l'étage ménagé au-dessus de l'entrée de l'enclos du manoir se diffuse à partir du XIII ${ }^{\mathrm{e}}$ siècle à tous les niveaux de l'aristocratie et n'est pas propre à l'espace Plantagenêt.

Ces monographies comme les autres contributions démontrent qu'il n'existe pas véritablement de programme spécifique à un état (laïc, ecclésiastique), à un niveau élitaire. En Angleterre, les grandes salles de plain-pied sous charpente avec ou sans bas-côtés, en pierre ou en bois, se rencontrent à tous les niveaux de l'aristocratie, voire chez les yeomen, les paysans aisés, pour les salles en bois (Meirion-Jones). Chez les élites, les exigences architecturales de logement et de démonstration du pouvoir, comme les modalités de restructuration et de mise au goût du jour, passent par les mêmes programmes qu'il ne faut pas envisager comme stéréotypés. Ils sont fonction de l'espace disponible dans l'enclos manorial et de la place dans la hiérarchie nobiliaire; en Angleterre (Dixon), les grandes salles de plain-pied à bascôtés seraient privilégiées par la haute aristocratie alors qu'aux niveaux inférieurs on préférerait la salle d'étage. Le prieuré de Moullins illustre cependant la prudence qui doit être de mise dans l'évaluation architecturale d'un site en fonction de son statut (Favre, Garrigou Grandchamp, Bouvet). Dans la première moitié du XIII ${ }^{\mathrm{e}}$ siècle et dans les années 1300-1340, l'enclos a été doté de deux grandes salles de plainpied sous charpente à une nef et deux bas-côtés, d'une ampleur qui pourrait être considérée comme inhabituelle pour un tel type de site $\left(350 \mathrm{~m}^{2}\right.$ restitués pour la plus récente). De la même façon, le logis de la ferme de Fresnay (milieu XIII ${ }^{\mathrm{e}}$ siècle), occupé par le fermier de cette dépendance de la mense épiscopale de Chartres, présente des dimensions, une structuration verticale (rez-de-chaussée et étage rési- 
dentiels largement éclairés, tourelle-échauguette d'angle) et des décors sculptés tout en ostentation (Audebrand).

Pour terminer on notera que c'était une bonne idée de donner vie à ces salles et chambres majoritairement nobles. Pour sa contribution, M. Nassiet convoque 33 inventaires nobles de tous niveaux majoritairement bretons ( $\mathrm{XV}^{\mathrm{e}}-\mathrm{XVI}^{\mathrm{e}}$ siècles) et il donne l'édition inédite de l'un d'entre eux (La Prévalaye, 1478). On sent l'hésitation de l'auteur dans sa démarche qui privilégie, et c'est d'un grand intérêt, les informations sur les dispositions architecturales de la maison que les scribes qui établissent l'inventaire dévoilent dans leur cheminement, alors que les meubles ne sont pas véritablement scrutés (nombre, dimensions, type de bois, localisation).

Ce compte rendu ne peut qu'être trop court pour présenter avec tous les détails nécessaires le grand intérêt de cet ouvrage pour notre évaluation de l'habitat noble $\mathrm{du} \mathrm{XI}^{\mathrm{e}}$ au XVe siècle dans toutes ses composantes et son évolution. Il côtoiera avec profit des ouvrages collectifs publiés sur les mêmes thèmes ces dernières années.

Marie CASSET

Michon, Cédric, Petris, Loris (dir.), Le cardinal Jean du Bellay. Diplomatie et culture dans l'Europe de la Renaissance, Rennes/Tours, PUR/Presses universitaires François-Rabelais, coll. « Renaissance », 2014, 390 p.

Jean du Bellay a de la chance : alors que la publication de sa riche correspondance a repris il y a quelques années après un quart de siècle d'interruption et qu'elle est désormais en voie d'aboutissement, il fait ici l'objet d'une ample publication pluridisciplinaire. Elle s'appuie sur l'ensemble de cette correspondance, mais mobilise également de nombreuses autres sources. Certains inédits sont d'ailleurs publiés en annexe dans l'ouvrage (p. 299-352). Celui-ci est également doté d'une riche illustration, avec un cahier couleur, d'une ample bibliographie (où on déplore juste quelques doublons) et d'un index des personnes.

Les dix-huit contributions du livre cherchent à cerner l'ensemble des aspects d'une personnalité et plus précisément d'une longue carrière, qui combine service du roi, en particulier diplomatique, engagement au sein de l'Église catholique, pratique littéraire et mécénat artistique. Ces diverses facettes mobilisent autour de Jean du Bellay des réseaux familiaux, politiques, cléricaux et culturels dont l'imbrication est au cœur de l'ouvrage, sur un espace qui dépasse largement le royaume de France. Si son engagement concerne en priorité l'Angleterre, l'Allemagne et l'Italie, il est révélateur de voir que sa correspondance fait référence, même si c'est pour ironiser sur leur caractère reculé, aussi bien à Calicut qu'au Canada. Bien plus proches de lui sont finalement les hommes de l'Antiquité, à laquelle il voue une véritable passion et dont il collectionne les reliques et pratique assidûment les textes.

Jean du Bellay est issu d'une famille de moyenne noblesse dont l'entrée au service d'un prince de stature internationale, le roi René, puis du roi de France, assure la réussite au cours du XVe siècle (L. Bourquin). Bénéficiant, comme ses frères, d'une éducation humaniste soignée, précoce pour ce milieu, ainsi que d'appuis haut placés (les Vendôme, Montmorency, sans doute Marguerite de Navarre), il entre dans les cercles du pouvoir dans les années qui suivent Pavie et entame aux côtés de son frère aîné Guillaume une carrière diplomatique tant du côté anglais (D. Potter) qu'allemand (T. Niklas). Les réseaux Du Bellay, imprégnés d'évangélisme (G. Alonge), luttent avant tout contre la puissance impériale, et pour ce faire tentent de négocier des compromis politico-religieux entre les autres puissances pour mieux isoler 